\title{
Comportamento ingestivo de novilhos de corte sob diferentes alturas de pastejo do capim Tanzânia ${ }^{1}$
}

\section{Ingestive behavior of steers grazing under different heights of Tanzânia grass}

\author{
Rondineli Pavezzi Barbero ${ }^{2 *}$; Marco Aurélio Alves de Freitas Barbosa ${ }^{3}$; \\ Letícia Maria de Castro ${ }^{4}$; Edson Luis de Azambuja Ribeiro ${ }^{3,1}$; Ivone Yurika \\ Mizubuti ${ }^{3,1}$; Fernando Luiz Massaro Júnior ${ }^{5}$; Leandro das Dores Ferreira da Silva ${ }^{3}$
}

Resumo

\begin{abstract}
Foi avaliado o comportamento ingestivo de bovinos em quatro alturas de pastejo do capim Tanzânia. Foram utilizados 12 piquetes de um hectare cada, com três repetições para cada altura, utilizando três bovinos nelore, machos por piquete, mais reguladores de altura. Foram observados horários e tempo diurno de pastejo, ruminação e ócio, taxa de bocados e ruminações, duração dos ciclos de pastejo e ruminação, além de serem colhidas amostras de forragem ao nível do solo e por simulação de pastejo para avaliar possível seletividade de forragem pelos animais. O delineamento foi inteiramente casualizado, e foi realizada análise de variância, sendo que nas variáveis contínuas aplicaram-se a regressão, e nas variáveis discretas, testes de médias $(\mathrm{P}<0,05)$. A taxa de bocados apresentou comportamento linear crescente conforme aumento da altura do dossel, enquanto que nas menores houve maior dispêndio de tempo na busca por massa de forragem. O tempo de pastejo foi linear decrescente conforme aumento da altura do dossel, mas o contrário foi observado para ruminação. Em todas as alturas foi observada seletividade de forragem pelos animais.
\end{abstract}

Palavras-chave: Ócio, ruminação, taxa de bocado

\begin{abstract}
Ingestive behavior of steers grazing in four heights of Tanzânia grass was evaluated. 12 paddocks of one hectare each, with three replicates for each height, and three males Nelore cattle, per paddock, more regulators at the time, were used. Time and time daytime grazing, ruminating and resting, rate of eating and rumination, length of grazing cycles and rumination, were observed. Forage samples were collected at ground level and grazing simulation to assess possible selectivity. The experimental design was completely randomized, and analysis of variance was performed for continuous variables to regression procedures, and discrete variables in tests of means $(\mathrm{P}<0.05)$. The highest heights had high bit rates, while the smallest was higher expenditure of time in search for forage mass. Grazing time decreased linearly as increased the canopy height, however the opposite was observed for rumination. At all canopy heights was observed selectivity of forage for the animals.
\end{abstract}

Key words: Bit rate, idleness, rumination

1 Parte da dissertação de Mestrado em Ciência Animal, do primeiro autor, Universidade Estadual de Londrina, UEL, Londrina, PR.

${ }^{2}$ Zootecnista, Discente de Doutorado de Pós-graduação em Zootecnia da Faculdade de Ciências Agrárias e Veterinárias do Campus de Jaboticabal, UNESP, Jaboticabal, SP. E-mail: rondinelibarbero@zootecnista.com.br

${ }^{3}$ Profs. Drs. do Dept ${ }^{\circ}$ de Zootecnia,Universidade Estadual de Londrina, UEL, Cx. Postal 6001, 86001-970, Londrina, PR. ${ }^{3,1}$ Bolsista de Produtividade do CNPq. Londrina-Pr. E-mail: maafbarbosa@uel.br; elar@uel.br; mizubuti@uel.br; leandro@uel.br

${ }^{4}$ Zootecnista, Mestre em Ciência Animal, UEL, Londrina, PR. E-mail: leticiacastro@zootecnista.com.br

${ }^{5}$ Zootecnista, Discente de Doutorado do Programa de Pós-graduação em Ciência Animal, UEL, Londrina, PR. E-mail: massaro@ uel.br

* Autor para correspondência 


\section{Introdução}

A compreensão sobre os hábitos e horários de pastejo dos bovinos é fundamental para que ocorra bom aproveitamento das pastagens, e para elaborar estratégias de manejo dos pastos (ZANINE et al., 2006). O desempenho de bovinos de corte apresenta ampla relação com seu potencial ingestivo, onde a capacidade de colheita de forragem pelo animal pode ser influenciável pelas características do relvado, como massa de forragem disponível; relação entre lâminas foliares verdes, colmos + bainhas e material senescente (COMBELLAS; HODGSON, 1979; TREVISAN et al., 2004).

O tempo despendido na colheita de forragem pode influenciar nas demais atividades a serem realizadas, afetando os processos fisiológicos fundamentais como a ruminação, e assim comprometer o aproveitamento do alimento ingerido. A longa duração do tempo de pastejo indica a dificuldade do animal em satisfazer sua demanda de alimento (STOBBS, 1970; GONTIJO NETO et al., 2006).

Osruminantes podemalteraro seu comportamento ingestivo com a finalidade de minimizar os efeitos de condições alimentares desfavoráveis, mas nem sempre conseguem impedir a diminuição do consumo diário de forragem (HODGSON, 1990). A taxa de bocado dada pelo número de bocados por minuto é uma escala temporal diretamente relacionada à taxa de ingestão instantânea, podendo ser limitada pela condição morfológica do dossel. Pesquisas têm apontado a altura do dossel forrageiro como a variável que representa modificações na taxa de ingestão instantânea (REGO et al., 2006), além da seletividade na apreensão do alimento (GONTIJO NETO et al., 2006).

O objetivo deste trabalho foi o de avaliar o efeito de diferentes alturas do dossel forrageiro de capim Tanzânia sobre os parâmetros ingestivos: tempo diurno de pastejo, ruminação e ócio, e seletividade da forragem por novilhos de corte.

\section{Material e Métodos}

O experimento foi realizado no município de Cidade Gaúcha, Estado do Paraná, Brasil. Segundo a classificação de Köppen o clima da região é Cfa. Análises químicas do solo à camada arável apresentaram os resultados: $\mathrm{pH}$ em $\mathrm{H}_{2} \mathrm{O}: 6,03 ; \mathrm{Ca}^{2}$ : 1,74 $\mathrm{cmol} / \mathrm{dm}^{3} ; \mathrm{Mg}^{2}: 0,52 \mathrm{mmol}_{\mathrm{c}} / \mathrm{dm}^{3} ; \mathrm{K}+: 0,23$ $\mathrm{mmol} / \mathrm{dm}^{3} ; \mathrm{Na}+: 0,00 \mathrm{cmol} / \mathrm{dm}^{3} ; \mathrm{P}$ disponível: 2,36 $\mathrm{mg} / \mathrm{dm}^{3}$; CTC efetiva: $2,49 \mathrm{cmol} / \mathrm{dm}^{3}$; e Al: $0,00 \%$.

Aárea experimental foi de 12 hectares com relevo levemente ondulado, com capim Tanzânia, dividida em 12 piquetes de um hectare, sendo utilizados três piquetes para cada altura do dossel: 20; 40; 60 e $80 \mathrm{~cm}$, distribuídos aleatoriamente. O período experimental foi de outubro de 2010 a janeiro de 2011, sendo os três primeiros meses destinados ao estabelecimento das alturas pretendidas e adaptação dos animais à altura do pastejo. Para manutenção das alturas de pastejo foram utilizados três bovinos machos, nelore, com peso médio inicial de $340 \mathrm{~kg}$, castrados e vermifugados, por piquete. A altura do dossel era mensurada em 20 pontos aleatórios por piquete, com régua graduada, na curvatura das lâminas foliares superiores, e conforme a necessidade de ajuste para manutenção das alturas eram introduzidos ou retirados animais adicionais, conforme metodologia descrita por Mott e Lucas (1952).

A avaliação do comportamento foi utilizada pelo método direto de observação visual (HUGHES; REID, 1951). As observações foram realizadas após o período de adaptação dos animais, por dois observadores treinados por piquete. Foram realizados dois períodos de observação, com 12 horas contínuas de observação diária, das 7:00 às 19:00 horas. Não foi realizada observação no período noturno devido à dimensão da área experimental, pois mesmo com a utilização de equipamentos para visão noturna não foi possível total observação. Alem disto, a utilização de mais observadores por piquete ou a movimentação de pessoas poderia influenciar nos resultados. 
Acada 10 minutos, foram registradas as atividades de pastejo, tempo de ruminação e ócio. O tempo de pastejo representa o período em que o animal está apreendendo ou selecionando a forragem. O tempo de ruminação é o tempo gasto pelo animal na mastigação do material retornado do rúmen, onde a observação é realizada pelo movimento bocal. No tempo despendido ao ócio estão incluídos ingestão de água e atividades sociais.

A taxa de bocados e de ruminações, ciclo de pastejo e de ruminações foram determinados por meio da escolha aleatória de um animal por piquete. Procederam-se estimativas visuais, medindo-se o tempo que o animal leva para completar 20 bocados de apreensão (FORBES; HODGSON, 1985), em intervalos de 10 minutos sempre que fosse verificada atividade de pastejo, e estimada a taxa por minuto. $\mathrm{O}$ mesmo procedimento foi realizado para observação de ruminação. Após observação do comportamento ingestivo, foram colhidas 20 amostras de forragem por piquete em simulação de pastejo, e 20 amostras pelo método do quadrado amostrador $\left(0,25 \mathrm{~m}^{2}\right)$, rente ao nível do solo, e determinada a composição bromatológica seguindo a metodologia descrita por Mizubuti et al. (2009), para comparação da composição bromatológica entre os materiais colhidos.
O delineamento estatístico foi inteiramente casualizado, com três repetições por tratamento, e com um animal por piquete para ciclo de bocados, taxa de bocados, ciclo de ruminações e taxa de ruminações. Para o tempo de pastejo, ruminação e ócio cada animal era uma repetição. Foi realizada análise de variância, ao nível de significância de 5\%, e aplicados procedimentos de regressão. A variação das atividades no decorrer das horas do dia foi comparada pelo teste Tukey $(\mathrm{P}<0,05)$, assim como a comparação entre a composição bromatológica da forragem colhida por simulação de pastejo e da composição do dossel forrageiro.

\section{Resultados e Discussão}

As alturas reais médias do dossel forrageiro na ocasião das avaliações foram 19,0;41,0;65,0 e $85,0 \mathrm{~cm}$ para as alturas pretendidas de $20 ; 40 ; 60$ e $80 \mathrm{~cm}$, respectivamente. A temperatura máxima durante o período de observação comportamental foi de $30,5^{\circ} \mathrm{C}$ entre $13 \mathrm{~h} 00 \mathrm{~min}$ e $16 \mathrm{~h} 00 \mathrm{~min}$, e a mínima de $20,8^{\circ} \mathrm{C}$ ao amanhecer. A precipitação pluviométrica total no mês de observação foi de 102,2 mm, não havendo ocorrência de chuvas nos dias de observação. A massa de forragem disponível em função das alturas de pastejo pode ser observada na Tabela 1.

Tabela 1. Massa de forragem (kg de matéria seca por hectare) de lâminas foliares verdes (MLF); colmos+bainhas (MCB) e material senescente (MMS) em pastos de capim Tanzânia sob diferentes alturas de pastejo.

\begin{tabular}{|c|c|c|c|c|c|c|}
\hline \multirow{2}{*}{ Estrutura } & \multicolumn{4}{|c|}{ 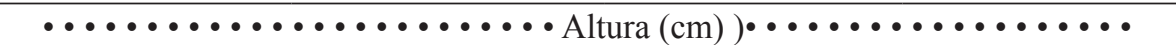 } & \multirow{2}{*}{$\mathrm{R}^{2}$} & \multirow{2}{*}{$\mathrm{P}$} \\
\hline & 20 & 40 & 60 & 80 & & \\
\hline MLF* & $3858,92 \pm 1933,35$ & $3415,03 \pm 1577,67$ & $4126,61 \pm 1971,57$ & $5132,83 \pm 2239,42$ & 0,62 & 0,049 \\
\hline $\mathrm{MCB}^{* *}$ & $5010,49 \pm 3368,09$ & $4574,15 \pm 2621,50$ & $5597,58 \pm 4171,31$ & $7615,50 \pm 6237,09$ & 0,72 & 0,048 \\
\hline MMS & $1076,38 \pm 1008,32$ & $926,64 \pm 964,46$ & $1060,75 \pm 1011,09$ & $754,02 \pm 1057,99$ & ns & ns \\
\hline
\end{tabular}

$* \mathrm{y}=3039,62+22,0725 \mathrm{x} ; * * \mathrm{y}=3489,82+44,1922 \mathrm{x} ; \mathrm{ns}=$ não significativo $(\mathrm{P}>0,05)$. MLF: massa de lâminas foliares; MCB: massa de colmos+bainhas; MMS: massa de material senescente.

Fonte: Elaboração dos autores. 
O tempo de duração do ciclo de bocados em minutos $(8,64 \pm 5,27)$ e da taxa de ruminações $(53,78$ $\pm 10,78)$ não diferiu significantemente em função da altura do dossel, indicando que o animal utilizou outro mecanismo de compensação à diferença entre disponibilidade de forragem e às alturas de dossel os tratamentos. A duração do ciclo de ruminações apresentou efeito quadrático, onde a altura de 44 cm apresentou o maior tempo de duração do ciclo, e posterior redução conforme aumento da altura do dossel, possivelmente em conseqüência do maior volume ingerido nestas condições. A taxa de bocados por minuto apresentou comportamento linear crescente com a altura do dossel forrageiro (Figura 1).

Figura 1. Duração média do ciclo de ruminações (CR) e taxa de bocados por minuto (TB) em bovinos de corte, em função das alturas de pastejo em capim Tanzânia.

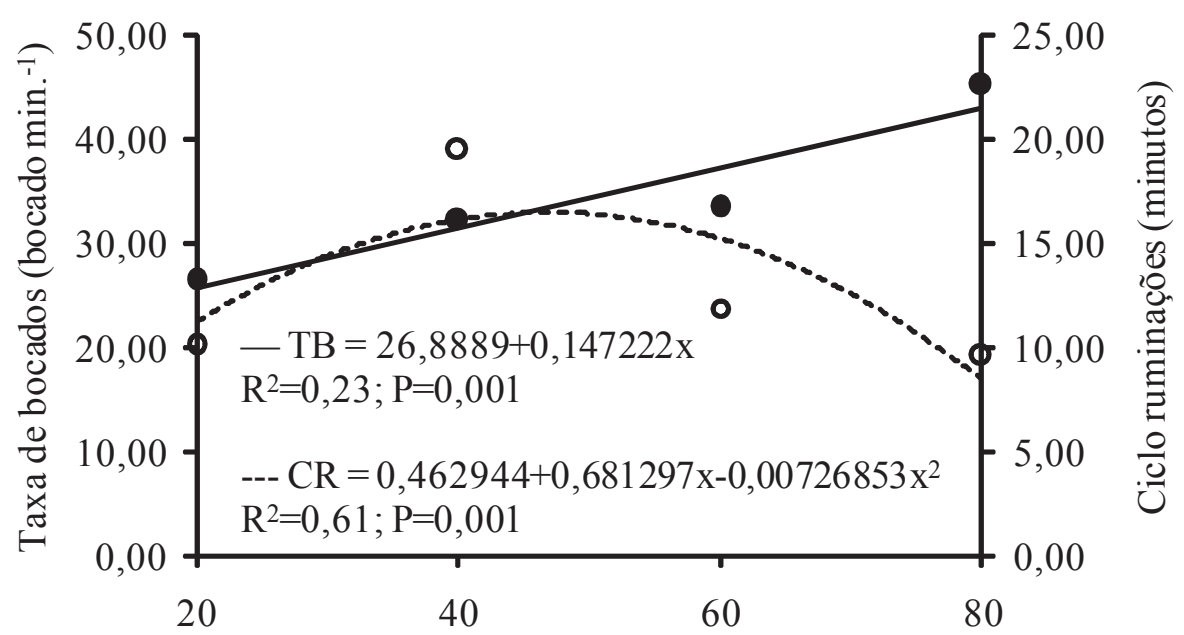

Altura (cm)

Fonte: Elaboração dos autores.

Foi observado que nas condições de maior altura do dossel forrageiro não ocorreu necessidade de locomoção do animal até a próxima touceira para o bocado seguinte, assim como não era necessário despender tempo na escolha das lâminas foliares a serem apreendidas, devido à maior oferta deste material, enquanto que nas menores alturas ocorreu a necessidade de locomoção do animal e seleção de touceiras com disponibilidade de lâminas foliares verdes, e ainda assim, estas em menor quantidade.

Segundo Zanine et al. (2006), em pastos com menores ofertas de lâminas foliares verdes os animais tendem a ser mais seletivos, resultando na alteração dos mecanismos do comportamento em pastejo para compensação. Esse dispêndio de tempo na procura e seleção por material a ser ingerido nas menores alturas resultou em maior intervalo entre bocados, e consequentemente em menor taxa de bocados por minuto nas menores alturas do dossel.

O período diário despendido em pastejo apresentou resposta linear negativa em função das alturas de pastejo (Figura 2), resultado contrário ao observado no tempo diário de ruminação, onde o comportamento foi linear positivo (Figura 2). A longa duração do tempo diário de pastejo pode ser um indicativo de que o animal está apresentando dificuldade de suprir sua demanda nutricional, mesmo quando grandes quantidades de forragem 
estão disponíveis (STOBBS, 1970). A densidade volumétrica exerce influencia importante sobre o volume do bocado, onde em pastos de maior densidade acarretam no aumento do tamanho do bocado (FLORES et al., 1993; REGO et al., 2006). Neste contexto, nos pastos de menor altura os animais aumentaram o tempo diário de pastejo como forma de compensar a ingestão de forragem, porém, ainda assim é bastante provável que o volume do bocado foi reduzido em relação aos pastos de maior altura.

Figura 2. Tempo diário de pastejo (TP) e de ruminação (TR) de novilhos de corte em pastos de capim Tanzânia em função das alturas de desfolha.

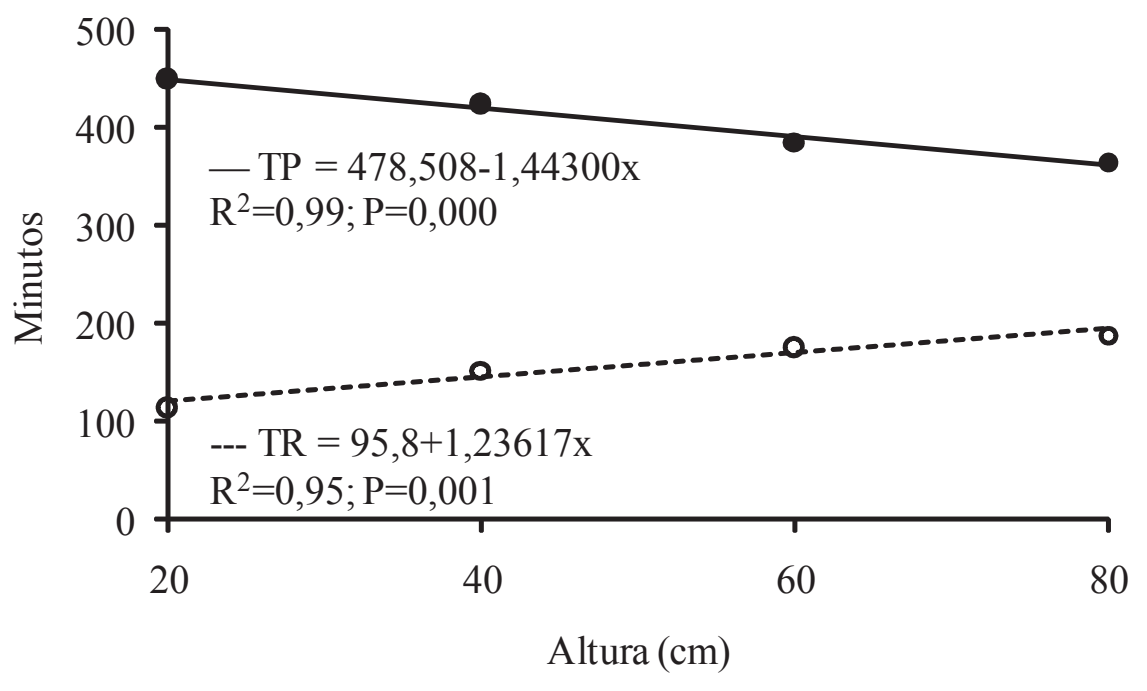

Fonte: Elaboração dos autores.

Nos pastos manejados de 20 a $60 \mathrm{~cm}$, foi observado aumento significativo na atividade de pastejo após 10h00min (Figura 3). Trevisan et al. (2004) também observaram maior atividade de pastejo no período vespertino em novilhos de corte pastejando aveia preta e azevém com níveis distintos de lâminas foliares verdes. Em alturas mais elevadas do dossel $(80 \mathrm{~cm})$, os picos de pastejo foram observados após as 16h00min (Figura 3), indicando que a maior massa de forragem disponível nesta condição permitiu aos animais realizarem picos de pastejo nos horários menos quentes do dia, com menor tempo diário de pastejo e assim, provavelmente atingindo sua demanda diária de ingestão de matéria seca.
O conhecimento dos horários de pastejo permite a realização de manejos de forma muito mais adequada. O fornecimento de suplementos, por exemplo, deve ser realizado fora dos horários de pico de pastejo (CABRAL; ZERVOUDAKIS; COPPEDÊ, 2008), evitando vícios de consumo ou ainda o efeito substitutivo, maximizando o consumo voluntário (PEREIRA et al., 2003). Os presentes resultados apontam que, em pastos de capim Tanzânia entre 20 e $60 \mathrm{~cm}$ sem a presença de sombreamento, as atividades de manejo devem ser realizadas antes das $10 \mathrm{~h} 00 \mathrm{~min}$, enquanto que em pastos de altura superior a $60 \mathrm{~cm}$, este horário pode se estender, devido ao fato de que a maior massa de forragem disponível permite que o animal apresente menor tempo diário de pastejo. 
Não houve diferença significativa quando comparados horários de ruminação nos pastos de 20 e $80 \mathrm{~cm}$, porém, nos pastos de 40 e $60 \mathrm{~cm}$ os picos de ruminação ocorreram pela manhã, entre 7 h00min e 10h00min. Não havia existência de sombra nos piquetes, fator este que possivelmente foi determinante nas atividades desenvolvidas pelos animais, como a preferência pela ruminação nos horários mais frescos do dia em algumas alturas de manejo. Pastos mais altos (60 e $80 \mathrm{~cm}$ ) não apresentaram diferença entre as horas do dia quanto ao ócio dos animais, demonstrando equilíbrio desta atividade ao longo do dia, porém, em pastos mais baixos (20 e $40 \mathrm{~cm}$ ) pôde ser observado ócio dos animais entre $7 \mathrm{~h} 00 \mathrm{~min}$ e $10 \mathrm{~h} 00 \mathrm{~min}$.

Figura 3. Atividades de pastejo (PAST) ruminação (RUMI) e ócio (OCIO) em bovinos de corte em pastos de capim Tanzânia sob $20 \mathrm{~cm}(\mathrm{~A}) ; 40 \mathrm{~cm}(\mathrm{~B}) ; 60 \mathrm{~cm}$ (C) e $80 \mathrm{~cm}$ (D) de altura em função das horas do dia.

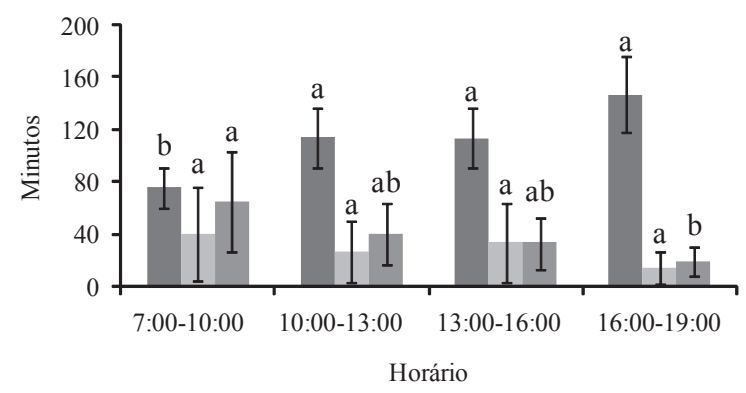

A)

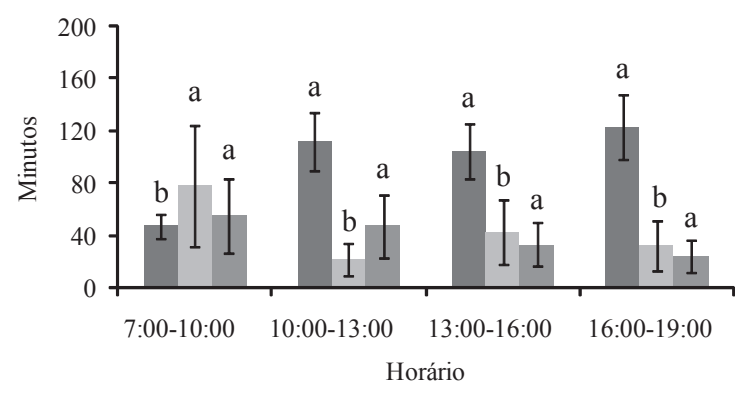

C)

$$
\text { - PAST } \approx \text { RUMI } \approx \text { OCIO }
$$

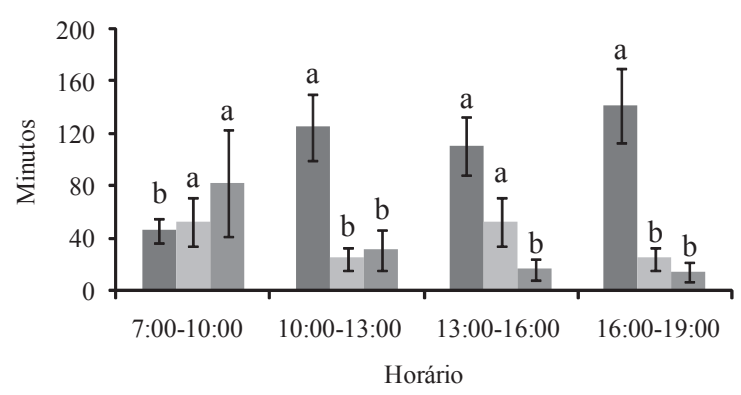

B)

$\because \mathrm{PAST} \backsim \mathrm{RUMI} \backsim \mathrm{OCIO}$

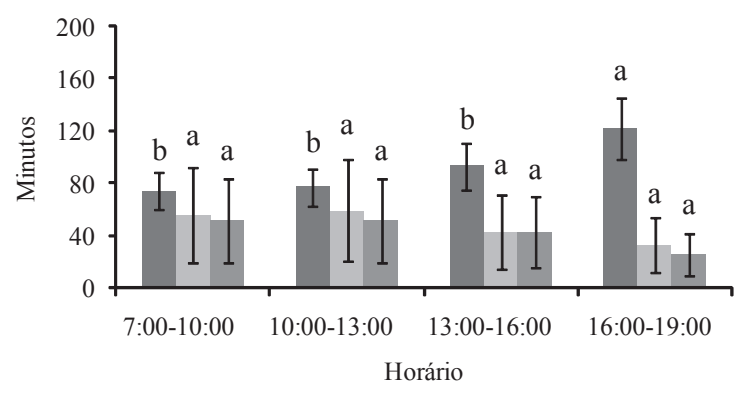

D)

Letras comparam os períodos, que quando iguais não diferem pelo teste de Tukey $(\mathrm{P}<0,05)$ na mesma variável.

Fonte: Elaboração dos autores.

A diferença das estruturas da planta forrageira quanto à composição bromatológica como proteína bruta e fibra em detergente neutro, causa efeito de seletividade pelo animal, buscando porções de melhor interesse nutricional. Esta situação ainda é agravada quando existe baixa disponibilidade de forragem (ZANINE et al., 2006). Em todas as alturas de pasto foram encontradas diferenças significativas entre a concentração de $\mathrm{MS}, \mathrm{PB}$, FDN, FDA e hemicelulose das amostras colhidas por simulação de pastejo ou rente ao solo, exceto para FDN na altura de $40 \mathrm{~cm}$, indicando possível equilíbrio entre a concentração das frações fibrosas da forrageira nesta altura, impossibilitando a seleção nestas condições (Tabela 2). 
A fibra em detergente neutro (FDN) é um fator dietético bastante representativo do volume ocupado pelo alimento, sendo, portanto, inversamente relacionado à densidade energética. A FDN em dietas com elevada proporção de fração fibrosa preenche os espaços do rúmen-retículo, levando maior tempo do que os conteúdos celulares para deixar este compartimento utilizando-se de mecanismos de digestão, ruminação e passagem (PEREIRA et al., 2003). Trabalhos recentes têm revelado que quando os pastos são devidamente manejados, a forragem produzida apresenta pouca variação quanto ao valor nutritivo dentre diferentes espécies e cultivares de plantas forrageiras (DA SILVA; NASCIMENTO JUNIOR, 2007).

Tabela 2. Comparação entre a composição bromatológica de capim Tanzânia colhida por simulação de pastejo (CSP) e colheita total do dossel forrageiro (CDF) sob quatro alturas de desfolha por bovinos de corte. Valores em $\mathrm{g} / \mathrm{kg}$ de matéria seca (exceto matéria seca).

\begin{tabular}{|c|c|c|c|c|c|}
\hline & MS & PB & FDN & FDA & Hemi \\
\hline & \multicolumn{5}{|c|}{ 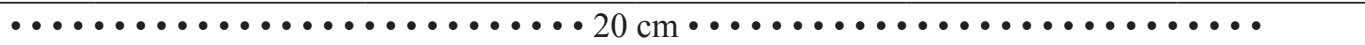 } \\
\hline CSP & $29,75 b$ & $11,12 \mathrm{a}$ & $70,13 b$ & $25,75 b$ & $43,37 \mathrm{a}$ \\
\hline $\mathrm{CDF}$ & $36,36 \mathrm{a}$ & $6,13 \mathrm{~b}$ & $76,16 \mathrm{a}$ & $40,83 \mathrm{a}$ & $35,33 b$ \\
\hline \multirow[t]{2}{*}{$\mathrm{CV}(\%)$} & 8,79 & 28,18 & 3,33 & 15,03 & 11,48 \\
\hline & \multicolumn{5}{|c|}{ 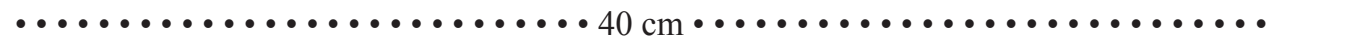 } \\
\hline CSP & $26,31 b$ & $10,07 \mathrm{a}$ & $71,52 \mathrm{a}$ & $30,93 b$ & $40,60 \mathrm{a}$ \\
\hline $\mathrm{CDF}$ & $37,60 \mathrm{a}$ & $7,03 b$ & $73,34 a$ & $37,89 \mathrm{a}$ & $35,44 b$ \\
\hline \multirow[t]{2}{*}{$\mathrm{CV}(\%)$} & 3,92 & 12,70 & 3,93 & 6,37 & 3,99 \\
\hline & \multicolumn{5}{|c|}{ 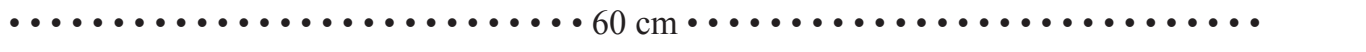 } \\
\hline CSP & $27,93 b$ & $13,00 \mathrm{a}$ & $70,06 \mathrm{~b}$ & $30,46 b$ & $35,60 \mathrm{a}$ \\
\hline $\mathrm{CDF}$ & $37,21 \mathrm{a}$ & $5,95 b$ & $75,04 \mathrm{a}$ & $40,07 \mathrm{a}$ & $35,67 b$ \\
\hline \multirow[t]{2}{*}{$\mathrm{CV}(\%)$} & 8,66 & 11,19 & 3,26 & 4,68 & 6,6 \\
\hline & \multicolumn{5}{|c|}{ 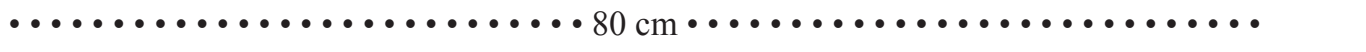 } \\
\hline CSP & $27,71 b$ & $11,53 \mathrm{a}$ & $71,75 b$ & $30,36 b$ & $40,89 a$ \\
\hline $\mathrm{CDF}$ & $34,57 \mathrm{a}$ & $6,74 b$ & $74,78 \mathrm{a}$ & $40,21 \mathrm{a}$ & $34,56 \mathrm{~b}$ \\
\hline $\mathrm{CV}(\%)$ & 5,6 & 7,15 & 3,37 & 3,85 & 5,61 \\
\hline
\end{tabular}

Letras iguais nas colunas (dentro da mesma altura) não diferem pelo teste de Tukey $(\mathrm{P}<0,05)$.

$\mathrm{CV}=$ coeficiente de variação. CSP: colheita em simulação de pastejo; CDF: colheita total do dossel forrageiro.

Fonte: Elaboração dos autores.

Embora ocorra seletividade das frações pastejadas pelos bovinos, outros fatores são mais impactantes sobre o desempenho animal. Segundo Da Silva (2011), a variação do valor nutritivo da forragem consumida pelos animais, fica em torno de 12 a $18 \%$ de proteína bruta, e de 60 a $70 \%$ de digestibilidade, valores estes que caracterizam boa qualidade do material. De acordo com o mesmo autor, as diferenças entre o desempenho animal são conseqüência, basicamente da quantidade de forragem ingerida, uma vez que a diferença em valor nutritivo é pequena, fato que realça a importância de conhecer e compreender como ocorre o consumo de forragem pelos animais em pastejo, e como ele é afetado pelas práticas de manejo utilizadas.

\section{Conclusões}

A taxa de bocados de bovinos de corte em pastos de capim Tanzania é diretamente proporcional a altura do dossel, enquanto que o tempo diário de pastejo é inversamente proporcional, na tentativa da compensação de ingestão de forragem nas menores alturas. Animais em pastos manejados a $80 \mathrm{~cm}$ 
de altura apresentaram pico de pastejo no final da tarde, enquanto nas demais alturas houve maior distribuição ao longo do dia. Em todas as alturas de manejo do pasto ocorreu seletividade das frações morfológicas de melhor interesse nutricional.

\section{Referências}

CABRAL, L. S.; ZERVOUDAKIS, J. T.; COPPEDÊ, C. M. Suplementação de bovinos de corte mantidos em pastagem de Panicum maximum cv. Tanzânia-1 no período das águas. Revista Brasileira de Saúde e Produção Animal, Salvador, v. 9, n. 2, p. 293-302, 2008.

COMBELLAS, J.; HODGSON, J. Herbage intake and Milk production by grazing dairy cows. The effects of variation in herbage mass and daily herbage allowance on short term trial. Grass and Forrage Science, Malden, v. 34, n. 4, p. 209-214, 1979.

DA SILVA, S. C. Intensificação da produção animal em pasto por meio do manejo do pastejo. In: SIMPÓSIO DE PRODUÇÃO ANIMAL A PASTO, 1., 2011, Maringá. Anais... Maringá: Eduem, 2011. p. 163-188.

DA SILVA, S. C.; NASCIMENTO JÚNIOR, D. Avanços na pesquisa com plantas forrageiras tropicais em pastagens: características morfofisiológicas e manejo do pastejo. Revista Brasileira de Zootecnia, Viçosa, MG, v. 36, p. 122-138, 2007.

FLORES, E. R.; LACA, E. A.; GRIGGS, T. C.; DEMMENT, M. W. Sward height and vertical morphological differentiation determine cattle bite dimensions. Agronomy Journal, Madison, v. 85, n. 3, p. 527-532, 1993.

FORBES, T. D. A.; HODGSON, J. Comparative studies of the influence of sward conditions on the ingestive behaviour of cows and sheep. Grass and Forage Science, Malden, v. 40, p. 69-77, 1985.

GONTIJO NETO, M. M.; EUCLIDES, V. B. P.; NASCIMENTO JUNIOR, D.; MIRANDA, L. F.; FONSECA, D. M.; OLIVEIRA, M. P. Consumo e tempo diário de pastejo por novilhos Nelore em pastagem de capim-tanzânia sob diferentes ofertas de forragem. Revista Brasileira de Zootecnia, Viçosa, MG, v. 35, n. 1, p. 60-66, 2006.
HODGSON, J. Grazing management: science into practice. 1990. $203 \mathrm{p}$.

HUGHES, G. P.; REID, D. Studies on the behavior of cattle and sheep in relation to utilization of grass. Journal of Agricultural Science, Cambridge, v.41, p. 350-355, 1951.

MIZUBUTI, I. Y.; PINTO, A. P.; PEREIRA, E. S.; RAMOS, B. M. O. Métodos laboratoriais de avaliação de alimentos para animais. Londrina: EDUEL, 2009. v. $1,228 \mathrm{p}$.

MOTT, G. O.; LUCAS, H. L. The design conduct and interpretation of grazing trials on cultivated and improved pastures. In: INTERNATIONAL GRASSLAND CONGRESS, 6., 1952, Pensylvania. Anais... Pensylvania: Pensylvania State College Press, 1952. p. 1380-1395.

PEREIRA, E. S.; DE ARRUDA, A .M. V.; MIZUBUTI, I. Y.; SILVA, L. D. F. Consumo voluntário em ruminantes. Semina: Ciências Agrárias, Londrina, v. 24, n. 1, p. 191196, 2003.

REGO, F. C. A.; DAMASCENO, J. C.; FUKUMOTO, N. M.; CÔRTES, C.; HOESHI, L.; MARTINS, E. N.; CECATO, U. Comportamento ingestivo de novilhos mestiços em pastagens tropicais manejadas em diferentes alturas. Revista Brasileira de Zootecnia, Viçosa, MG, v. 35, n. 4, p. 1611-1620, 2006.

STOBBS, T. H. Automatic measurement of the grazing time by dairy cows on tropical grass and legume pastures. Tropical Grassland, Sydnei, v. 4, n. 3, p. 237-244, 1970.

TREVISAN, N. B.; QUADROS, F. L. F.; SILVA, A. C. F.; BANDINELLI, D. G.; MARTINS, C. E. N.; SIMÕES, L. F. C.; MAIXNER, A. R.; PIRES, D. R. F. Comportamento ingestivo de novilhos de corte em pastagem de aveia preta e azevém com níveis distintos de folhas verdes. Ciência Rural, Santa Maria, v. 34, n. 5, p. 1543-1548, 2004.

ZANINE, A. M.; SANTOS, E. M.; PARENTE, H. N.; FERREIRA, D. J.; CECON. P. R. Comportamento ingestivo de bezerros em pastos de Brachiaria brizantha e Brachiaria decumbens. Ciência Rural, Santa Maria, v. 36, n. 5, p. 1540-1545, 2006. 